\title{
Genomic imprinting and genetic effects on muscle traits in mice
}

\author{
Stefan Kärst ${ }^{1 \dagger}$, Ali R Vahdatii ${ }^{2 \dagger}$ Gudrun A Brockmann ${ }^{1}$ and Reinmar Hager ${ }^{1 *}$
}

\begin{abstract}
Background: Genomic imprinting refers to parent-of-origin dependent gene expression caused by differential DNA methylation of the paternally and maternally derived alleles. Imprinting is increasingly recognized as an important source of variation in complex traits, however, its role in explaining variation in muscle and physiological traits, especially those of commercial value, is largely unknown compared with genetic effects.

Results: We investigated both genetic and genomic imprinting effects on key muscle traits in mice from the Berlin Muscle Mouse population, a key model system to study muscle traits. Using a genome scan, we first identified loci with either imprinting or genetic effects on phenotypic variation. Next, we established the proportion of phenotypic variation explained by additive, dominance and imprinted QTL and characterized the patterns of effects. In total, we identified nine QTL, two of which show large imprinting effects on glycogen content and potential, and body weight. Surprisingly, all imprinting patterns were of the bipolar type, in which the two heterozygotes are different from each other but the homozygotes are not. Most QTL had pleiotropic effects and explained up to 40\% of phenotypic variance, with individual imprinted loci accounting for $4-5 \%$ of variation alone.

Conclusion: Surprisingly, variation in glycogen content and potential was only modulated by imprinting effects. Further, in contrast to general assumptions, our results show that genomic imprinting can impact physiological traits measured at adult stages and that the expression does not have to follow the patterns of paternal or maternal expression commonly ascribed to imprinting effects.
\end{abstract}

\section{Background}

Genomic imprinting is increasingly seen as an important epigenetic source of phenotypic variation that may act in addition to or in conjunction with genetic sources of variation [1-3]. Imprinting occurs when identical alleles are expressed depending on whether they were inherited from the father or the mother, thus causing a parent-oforigin-specific gene expression pattern where either the paternal allele is expressed and the maternal allele is silenced (paternal expression; e.g. Igf2) or vice versa (maternal expression; e.g. Igf2R; [4]). Differential expression may be more common than complete silencing of one allele and expression of the other. At the molecular level, imprinting is caused by differential DNA methylation and histone modifications or non-coding RNAs

\footnotetext{
* Correspondence: reinmar.hager@manchester.ac.uk

${ }^{\dagger}$ Equal contributors

'Department for Crop and Animal Sciences, Humboldt-University Berlin, Berlin, Germany

Full list of author information is available at the end of the article
}

that, in mammals, is established early in embryogenesis [5]. At the phenotypic level, genomic imprinting is manifest in differences between reciprocal heterozygotes (which are genetically equivalent in a 2-allele system) as the only difference between these is the parent-of-origin of the two parental alleles [6].

Only about 100 imprinted genes have been formally described in mice (geneimprint.org, March 2012), yet significantly more imprinted loci have been described in quantitative genetic studies (e.g. [7-9]), several of which show complex interaction effects dependent on environmental factors $[1,10]$. It should be noted, however, the discovery of imprinting effects in studies using allele substitution estimation (of the paternally vs maternally derived allele) in F2 crosses (e.g. [7]) has been criticized for being biased toward the detection of spurious imprinting effects [11]. On the other hand, using DNA sequence characteristics and a machine learning approach, Luedi et al. [12] predicted that 600 of over 23,000 annotated genes are imprinted in the mouse. These quantitative 
genetic studies provide vital information on the location and effects of imprinted genes that may then be followed up in knock-out studies for functional description and evaluation. Perhaps even more important is that these studies illustrate that most imprinted gene effects do not occur in isolation but may show interactions with other genetic or environmental factors, thus establishing the biological network and pathways in which imprinted genes contribute to phenotypic variation. This can be achieved with knock-out studies only within the limited context of one or two loci.

The recognition that imprinting may be more common and important than previously thought has led to an expansion of studies in this field employing quantitative genetic approaches in addition to molecular studies. While early studies have focused on imprinted gene effects that occur during early development [13], more recent work has demonstrated that imprinting effects are not limited to early development $[7,9,14]$ nor are they limited to tissues involved in maternal / fetal interaction. We now know that imprinting can affect body composition traits, growth, behaviour $[8,15]$ as well as cognitive abilities in mice and humans [16]. In addition, studies have now started to estimate how much phenotypic variation is explained by variation at imprinted loci, an indication of how important imprinting may be as a source of variation [17]. For example, Wolf et al. [9] showed that pleiotropic imprinting effects can explain up to almost $6 \%$ of phenotypic variation alone.

Traditionally, research on imprinting has distinguished only two patterns of expression, namely paternal and maternal expression, and many data bases of imprinted genes follow this convention (e.g. geneimprint.org; http://igc.otago.ac.nz). However, more complex patterns have been discovered [9] with one of the notable examples being the callipyge phenotype described in sheep where one of the two heterozygotes shows muscular hypertrophy while the other three genotypes have normal appearance and do not differ from each other $[18,19]$. Although some pioneering work on imprinting was done in livestock (e.g. [7,20]), the importance of this key epigenetic mechanism for traits of commercial value is only beginning to be recognized in research efforts. However, few studies have been able to clearly separate maternal genetic effects from imprinting effects [21], which can cause the same phenotypic patterns [22], and have assessed how important imprinting is in explaining phenotypic variation compared to genetic sources of variation such as dominance and additive effects.

In this study, we have used a quantitative genetic approach to identify imprinted loci that modulate key obesity and muscle traits (body weight, body lean mass, body fat mass, lactate value, muscle glycogen content and muscle glycolytic potential) in mice derived from the Berlin Muscle Mouse Inbred (BMMI) strains. We first scanned the entire genome for QTL that show epigenetic (causing parent-of-origin phenotypic effects) as well as genetic effects (additive and dominance). Next, we determined whether parent-of-origin-specific effects are due to genomic imprinting or maternal genetic effects as only the former are true epigenetic effects. Finally, the proportion of phenotypic variation accounted for by additive, dominance and genomic imprinting effects was calculated. Our analysis enables us to investigate loci with pleiotropic effects and, at the same time, to investigate whether epigenetic or genetic effects are more important in explaining phenotypic variance in traits of interest.

\section{Results}

We discovered genomic imprinting effects located on chromosome 19 with a strong effect on glycolytic potential and content. The imprinting pattern was bipolar where the two heterozygotes are different but the two homozygotes are not, consistently for all affected traits (Figure 1). The bipolar pattern is also reflected in the absence of an additive genetic effect, which is required for either paternal or maternal expression [8]. This is somewhat surprising as one might have expected a parental expression pattern (either paternal or maternal expression) since these patterns are commonly assumed to be more prevalent. An additional imprinted locus was found for body weight on chromosome 12. Figure 1 shows two different patterns of bipolar imprinting expression. While body weight shows a pattern with a higher value for the maternal heterozygote $(B / A)$, glycolytic potential and glycogen content have higher values for the paternal heterozygote $(A / B)$.

A closer look at the genomic structure of the imprinted regions shows that the iQTL at $27.3 \mathrm{Mb}$ on chromosome 12 comprises an informative haplotype block of $8.2 \mathrm{Mb}$ (corresponding to $4.3 \mathrm{cM}$, [23]) between 26.6 and $34.8 \mathrm{Mb}$ where the alleles differ between BMMI806 and BMMI816. This region is well represented by linkage disequilibrium in the $F_{3}$ population with 0.4 and $3.9 \mathrm{cM}$ on each side of the marker at the peak IQTL position. The next informative regions are 12.7 and $14.2 \mathrm{Mb}$ proximal and distal of the peak marker, respectively, and are only in weak linkage disequilibrium with the peak iQTL marker. With regard to the iQTL on chromosome 19, the marker at the peak iQTL position at $28.9 \mathrm{Mb}$ is located in an informative genomic region of $1.8 \mathrm{Mb}$ (1.7 cM, [23]) between 27.5 and 29.3 Mb. Additional informative genomic regions are located between 24.2 and $24.5 \mathrm{Mb}$, and 35.2 and $35.5 \mathrm{Mb}$ which are $4.5 \mathrm{Mb}(4.2 \mathrm{cM})$ and $6.3 \mathrm{Mb}$ (5.9 cM) distant from the peak iQTL marker, respectively. However, the marker at $35.2 \mathrm{Mb}$ did not show 


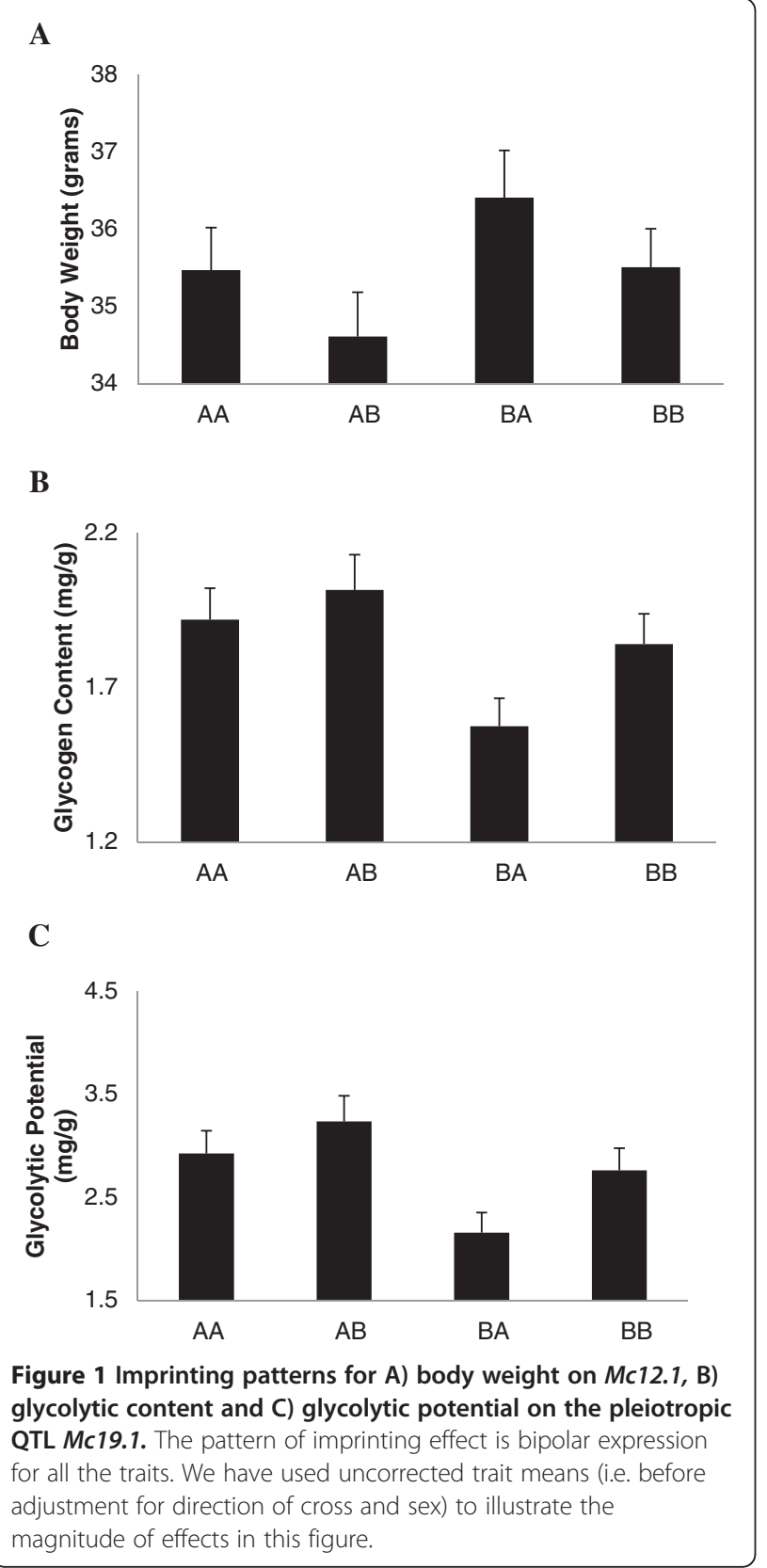

imprinting effects. Since the informative marker intervals on chromosomes 12 and 19 are small, we have high confidence in the peak locations and genotyping of additional markers would not improve the iQTL detection, neither in respect to position nor significance. Frequencies of all four genotype classes at the peak marker loci are balanced (range: 0.20 to 0.31 , Additional file 1: Table S1) so that test statistics are unlikely to be biased.

We detected a further seven QTL on different chromosomes affecting variation at all seven traits (Table 1). Most loci showed pleiotropic effects (e.g. Mc1.1, Mc2.1, Mc19.1), however, four loci affected a single trait only
(Mc6.1, Mc12.1, Mc12.2 and Mc17.1). Although we identified several loci that affected overall body weight and the correlated traits fat and lean mass (e.g. Mc1.1), two loci affected overall body weight only (Mc12.1 and Mc17.1) but not fat mass and lean mass (traits commonly associated with body weight). Six of the nine loci showed clear additive effects, followed by two imprinted QTL and one dominance locus. This pattern is evinced in the number of genetic effects for all traits: additive effects show up 16 times, imprinting effects nine times and dominance effects only five times (Table 1 ).

Next, we calculated the proportion of phenotypic variation explained by individual loci and their effects (Table 2). Because our QTL model is orthogonal we can add up the proportion explained by individual effects [17]. Table 2 also summarizes the pleiotropic QTL effects observed at most loci by giving the number of loci and their individual effects. Interestingly, variation for glycolytic potential and content are modulated only by genomic imprinting effects. It can be seen that our detected QTL explain up to $40 \%$ of phenotypic variation (fat mass). For the majority of traits, additive effects explain most of the variation. However, for body weight and muscle mass, dominance and imprinting effects contribute a significant proportion to the overall variation explained. The imprinted loci have relatively strong QTL effects, individually accounting for $4 \%$ to $4.7 \%$ of variation.

\section{Discussion}

The key result of this study is that genomic imprinting effects but not genetic variation affects glycolytic potential and glycogen content in the BMM mouse lines selected for divergent muscle traits. We discovered a further seven loci with additive and dominance effects that modulate variation in muscle and obesity traits. Overall, variation at our nine loci accounted for up to $40 \%$ of phenotypic variation, a considerable amount. There was a clear distinction between the three loci that exert strong pleiotropic effects (Mc1.1, Mc2.1 and Mc19.1) and those that affect only one trait (Mc6.1, Mc12.1, Mc12.2 and Mc17.1).

The results of this study clearly further strengthen the evidence for the role of genomic imprinting at later life stages (e.g. $[17,25])$ : the imprinting effects found here were manifest at 10 weeks of age, well into adulthood for these mice. Moreover, the patterns at the two imprinted loci were not the traditional paternal or maternal expression pattern. Another deviation from previously held views is that imprinted genes are predicted to mainly affect resource transfer between mother and utero (although Haig's conflict model upon which this view rests has always been more general [26]). However, here we can demonstrate that genomic imprinting affects physiological traits, in line with recent research 
Table 1 Identified loci with effect scores

\begin{tabular}{|c|c|c|c|c|c|c|c|c|}
\hline QTL & Trait & Pos Mb & Pos cM & C.I cM & $\mathrm{mLOD}$ & $a \mathrm{LOD}$ & $d \mathrm{LOD}$ & iLOD \\
\hline \multirow[t]{7}{*}{ Mc1.1 } & Body weight & 34.6 & 13.3 & $13.33-44.4$ & 4.91 & 4.89 & 0.88 & 0.45 \\
\hline & Lean mass & & & $13.33-44.4$ & 3.6 & 3.53 & 1.03 & 0.24 \\
\hline & Glycolytic potential & & & 13.33 & 0.92 & 0.56 & 1.68 & 0.09 \\
\hline & Fat mass & & & $13.33-40.2$ & 2.28 & 3.21 & 0.81 & 0.13 \\
\hline & M. quadriceps & & & 13.33 & 1.56 & 2.02 & 0.31 & 0.39 \\
\hline & M. longissimus & & & $13.33-26.7$ & 1.13 & 1.87 & 0.16 & 0.09 \\
\hline & Muscle mass & & & 13.33 & 1.44 & 2.17 & 0.21 & 0.02 \\
\hline \multirow[t]{5}{*}{ Mc2.1 } & M. longissimus & 82.0 & 49.1 & $49.1-54.1$ & 3.08 & 3.55 & 1.23 & 1.23 \\
\hline & Lean mass & & & $49.1-54.1$ & 2.86 & 3.53 & 1.47 & 0.96 \\
\hline & M. quadriceps & & & $49.1-54.1$ & 4.02 & 4.59 & 1.06 & 1.15 \\
\hline & Body weight & & & $49.1-54.1$ & 1.86 & 2.72 & 1.24 & 0.45 \\
\hline & Muscle mass & & & $49.1-54.1$ & 4.35 & 4.94 & 1.46 & 1.26 \\
\hline Mc6.1 & M. longissimus & 12.1 & 5.3 & $1.8-11.2$ & 3.30 & 3.39 & 0.51 & 1.36 \\
\hline \multirow[t]{2}{*}{ Mc8.1 } & M. longissimus & 79.4 & 36.3 & $33.1-36.3$ & 2.74 & 3.69 & 1.14 & 0.20 \\
\hline & Muscle mass & & & $33.1-36.3$ & 2.94 & 3.97 & 1.12 & 0.09 \\
\hline Mc12.1 & Body weight & 27.3 & 9.7 & $9.7-28.7$ & 2.24 & 0.21 & 0.34 & 3.30 \\
\hline Mc12.2 & Fat mass & 91.4 & 43.9 & $51.4-61.2$ & 2.17 & 3.30 & 0.11 & 0.01 \\
\hline \multirow[t]{2}{*}{ Mc15.1 } & Muscle mass & 10.3 & 58.7 & 58.7 & 2.57 & 0.19 & 3.32 & 0.13 \\
\hline & M. quadriceps & & & $50.4-58.7$ & 3.29 & 0.41 & 4.08 & 0.01 \\
\hline Mc17.1 & Body weight & 77.6 & 48.1 & $58.1-60.9$ & 2.87 & 3.63 & 0.07 & 0.88 \\
\hline \multirow[t]{7}{*}{ Mc19.1 } & Glycolytic potential & 28.9 & 23.5 & 23.5 & 3.91 & 0.49 & 1.30 & 3.33 \\
\hline & Body weight & & & $23.53-29.8$ & 1.77 & 1.23 & 0.09 & 1.93 \\
\hline & Glycogen content & & & 23.53 & 2.60 & 0.43 & 0.50 & 2.94 \\
\hline & Lean mass & & & 23.53 & 2.21 & 1.5 & 0.01 & 2.20 \\
\hline & M. longissimus & & & $23.53-29.8$ & 1.91 & 0.39 & 0.06 & 2.78 \\
\hline & Muscle mass & & & $23.53-29.8$ & 2.07 & 0.40 & 0.05 & 2.82 \\
\hline & M. quadriceps & & & $23.53-38.8$ & 1.69 & 0.39 & 0.54 & $\begin{array}{l}1.87 \\
\end{array}$ \\
\hline
\end{tabular}

M. quadriceps is the summed up mass of the two M. quadriceps, M. longissimus is the mass of the two M. Longissimus, muscle mass is the sum of the two M. longissimus and the two M. quadriceps, fat mass is the body composition's fat mass and lean mass is the body composition's lean mass, glycogen content is measured from M. longissimus. Pos Mb is the physical position of the marker in Mb and Pos CM is the position of marker in centiMorgans. LOD scores in bold are significant. Confidence intervals (C.I.) are given in CM using a one LOD drop following Lander \& Botstein [24].

Table 2 Proportion of phenotypic variance explained by all QTL for each trait

\begin{tabular}{|c|c|c|c|c|c|c|c|c|}
\hline Trait & Loci & $a$ effects & $d$ effects & $i$ effects & $\mathrm{R}^{2} a$ & $\mathrm{R}^{2} d$ & $\mathrm{R}^{2} i$ & Total $\mathrm{R}^{2}$ \\
\hline Body weight & 8 & 7 & 0 & 1 & 26.35 & 0 & 2.71 & 29.06 \\
\hline Muscle mass & 7 & 6 & 1 & 0 & 19.08 & 4.03 & 0 & 23.11 \\
\hline Fat mass & 5 & 4 & 0 & 0 & 39.36 & 0 & 0 & 39.36 \\
\hline Lean mass & 7 & 7 & 0 & 0 & 15.28 & 0 & 0 & 15.28 \\
\hline Glycolytic potential & 1 & 0 & 0 & 1 & 0 & 0 & 4.66 & 4.66 \\
\hline M. quadriceps & 5 & 4 & 1 & 0 & 6.15 & 1.25 & 0 & 7.40 \\
\hline M. longissimus & 5 & 5 & 0 & 0 & 14.60 & 0 & 0 & 14.60 \\
\hline Glycogen content & 1 & 0 & 0 & 1 & 0 & 0 & 4.05 & 4.05 \\
\hline
\end{tabular}

Column two specifies the total number of QTL detected for each trait. Columns 3, 4 and 5 give the QTL with their respective additive, dominance and imprinting effects., $R^{2} a, R^{2} d, R^{2} i$ and Total $R^{2}$ denote to the phenotypic variance explained by the, additive effect, dominance effect, imprinting effect and main QTL effect, respectively. 
showing imprinting effects on body composition and other complex traits $[2,8]$.

The confidence regions of our imprinted QTL do not encompass any known imprinted genes (cf. geneimprint. com; March 2012). However, RNA-seqence analysis of brain tissues by Gregg and colleagues [27] revealed a much greater number of loci that show parent-of-origin specific differences in gene expression. Bearing in mind that these data are from different tissues compared to our phenotypes, we do find that our two imprinted loci (Mc12.1 and Mc19.1) are within regions identified by Gregg and colleagues in the hypothalamus and the medical prefrontal cortex (Additional file 2: Table S2). However, the expression patterns for our loci are clearly bipolar whereas Gregg and colleagues only distinguish parental expression patterns. For our tissues, we thus regard our iQTL as novel imprinted loci, which warrant further investigation both in model systems and at the molecular level. However, Mc1.1 showing additive effects for multiple muscle traits is within the confidence region of previously found additive QTL for body weight, lean mass and fat mass [28], and also in line with the QTL on chromosome 1 for growth found by Brockmann et al. [29]. The finding of bipolar imprinting effects on glycogen content and glycogen potential together with the absence of other genetic effects is intriguing, showing that imprinting can play an important role in modulating physiological traits of importance in livestock breeding. Glycogen content and glycolytic potential are determinant factors in meat quality [30], affecting the ultimate $\mathrm{pH}$ of meat, which, in turn, affects other quality traits such as water holding capacity, incidence of spoilage [31,32] and meat quality [33]. A mutation in the PRKAG3 gene in Hampshire pigs, for example, causes high glycogen content in skeletal muscle, which has beneficial effects on meat content but detrimental effects on processing yield [34].

Results of our analysis of the proportion of phenotypic variance explained by additive, dominance and imprinting effects contrasts with results of weight traits in a different population generated from mouse lines divergent for body weight at day 60 [17]. In this study, most traits were affected by additive, dominance and imprinting effects with additive effects explaining most of the variation, followed by dominance and imprinting effects. By contrast, the genetic architecture of muscle and fat traits described in the present study is different, although clearly a large part of body weight. Here, few dominance effects (5) were detected, followed by imprinting effects (9), and 16 additive effects across all traits. Moreover, genomic imprinting was the only significant source of variation at glycogen content and glycolytic potential, explaining $\sim 4.5 \%$ of variation. This result warrants further investigation, in particular, whether selection for muscle traits may have resulted in selection at imprinted loci. Given that the imprinting pattern is bipolar, i.e. there is no additive effect (difference between homozygotes), it will be intriguing to explore how selection may have resulted in such an imprinting pattern or whether alternative explanations need to be invoked. This point seems particularly relevant to livestock production in which special breeding schemes are often used to benefit from heterosis effects and this paper contributes to encircle relevant regions for commercially important traits.

\section{Conclusion}

Our results show that variation in key physiological traits such as glycogen content is modified by parent-oforigin-dependent gene expression or genomic imprinting but not by genetic variation. These results further demonstrate that imprinting effects are not limited to early developmental phenotypes nor that imprinting occurs predominantly as paternal or maternal expression.

\section{Methods}

Animals

For this study, we used mice of the Berlin Muscle Mouse (BMM) population, which has been selected for high body weight and muscle mass, primarily in order to investigate the selective mechanisms in livestock breeding [35]. The selection history of BMM has been described previously [28]. We used the BMMI806 and BMMI816 lines, which are hyper-muscular but do not carry the Mstn ${ }^{\text {Cmptdl1Abc }}$ mutation. Two pairs of the Berlin Muscle Mouse inbred lines BMMI806 and BMMI816 were crossed reciprocally to generate the $F_{1}$ generation [28]. 94 $\mathrm{F}_{2}$ animals were produced that were then randomly mated [36] to produce $345 \mathrm{~F}_{3}$ animals. For the analysis of imprinting effects, we used all $94 \quad F_{2}$ animals and $331 \mathrm{~F}_{3}$ animals out of 345 for our QTL analysis because of genotyping errors in some individuals.

\section{Husbandry}

All experimental protocols were approved by the German Animal Welfare Authorities (approval no. G0405/ $08)$. The animals were maintained under standard conditions $\left(22 \pm 2^{\circ} \mathrm{C}\right.$ temperature; 12:12 hours light:dark cycle). Two to four animals of the same sex were put in cages with ad libitum access to food and water. The animals were fed a standard diet (Altromin standard breeding diet no. 1314 TPF, Lage, Germany) until they were 70 days old. This diet was composed of $27.0 \%$ crude protein, $5.0 \%$ crude fat, $4.5 \%$ crude fibre, $6.5 \%$ crude ash, $50.5 \%$ nitrogen free extract (starch and sugar), vitamins, trace elements and minerals $(2988 \mathrm{kcal} / \mathrm{kg}$ metabolizable energy; thereof $27.0 \%$ energy from proteins, $13.0 \%$ from fat and $60.0 \%$ from carbohydrates). 


\section{Phenotypic measures}

The mice, at 71 days of age, were anaesthetized by isoflurane and sacrificed after two hours of fasting. The Musculus longissimus (ML) and Musculus quadriceps (MQ) were dissected and weighed, and muscle mass (MM) was recorded as summed muscle weight of left and right $M$. longissimus and left and right $M$. quadriceps. The right muscles were promptly frozen in liquid nitrogen and then stored at $-80^{\circ} \mathrm{C}$. Carcasses were kept at $6{ }^{\circ} \mathrm{C}$ and $\mathrm{pH}$ values were measured within the $\mathrm{M}$. biceps femoris at 1 and 24 hours post mortem (ebro PHT 810, Ingolstadt, Germany). The glycolytic potential was measured from glycogen content and lactate content [37]. We adjusted the phenotypes for sex and directionof-cross (Parental Grand Mother) reducing variance not attributed to QTL. Values for total fat and total lean mass were measured by quantitative magnetic resonance (QMR) analysis, using the EchoMRI whole body composition analyser (Echo Medical Systems, Houston, Texas, USA [38,39]). After the two-hour fasting, before dissection, blood glucose levels were measured. We measured the muscle glycogen content colorimetrically in the right $\mathrm{M}$. longissimus (GOD/PAP method 'Glucose liquicolor' by Human, Wiesbaden, Germany) following Barham and Trinder [40]

\section{Genotyping}

The two parental lines BMM806 and BMMI816 were generated from the same founder population that had been selected for high muscularity over many generations. Since we genotyped both lines with the Mouse Diversity SNP Array [41], comprising 623,124 singlenucleotide polymorphisms (SNPs), we could identify haplotypes that were identical or differed between the two lines. Using the high dense SNP information, we selected 164 informative markers on all chromosomes, except Y. Marker distances in genomic regions that differed between the two parental lines were below $10 \mathrm{Mb}$, which was about $5 \mathrm{cM}$ (Additional file 3: Figure S1) [23]. A higher marker density would not lead to a higher mapping resolution in this pedigree [42]. Intervals larger than $10 \mathrm{Mb}$ did not contain informative markers and thus could not add information to the linkage analysis.

All animals of the $F_{2}$ and $F_{3}$ generations were genotyped at KBiosciences (Hoddesdon, U.K.). Genotypes were checked for errors and genetic distances with RQTL (http://cran.r-project.org/web/packages/RQTL/ index.html). Genotype frequencies at every marker locus are given in Additional file 1: Table S1. Although imprinted genes on the $\mathrm{X}$ chromosome have been reported, the imprinting analysis for our model is unresolved at present. The conversion of the physical map into a genetic map was performed using "Mouse Map Converter" software from the Jackson Laboratory.

\section{QTL analysis}

The data analysis for this paper was conducted using SAS 9.1 (SAS Institute, Cary, NC) and SPSS (SPSS Inc., Chicago IL) and PedPhase (version 2.0). We reconstructed haplotypes using Pedphase [43] to produce a set of unordered haplotypes for the $\mathrm{F}_{2}$ generation and a set of ordered (by allelic parent-of-origin) haploytpes for the $\mathrm{F}_{3}$. We distinguish four ordered genotypes denoted $A A, A B, B A, B B$ (paternal / maternal allele) with the $A$ allele originating from BMMI806 and the $B$ allele from BMMI816. In a first step we assigned the four ordered genotypes at the marker loci additive $(a)$, dominance $(d)$, and parent-of-origin (i) genotypic index scores following Wolf et al. [9].

$$
\left[\begin{array}{l}
\overline{A A} \\
\overline{A B} \\
\overline{B A} \\
\overline{B B}
\end{array}\right]=\left[\begin{array}{cccc}
1 & 1 & 0 & 0 \\
1 & 0 & 1 & 1 \\
1 & 0 & 1 & -1 \\
1 & -1 & 0 & 0
\end{array}\right]\left[\begin{array}{l}
r \\
a \\
d \\
i
\end{array}\right]
$$

This can be solved to yield estimates of the genotypic values

$$
\left[\begin{array}{l}
r \\
a \\
d \\
i
\end{array}\right]=\left[\begin{array}{c}
\frac{\overline{A A}}{2}+\frac{\overline{B B}}{2} \\
\frac{\overline{A A}}{2}-\frac{\overline{B B}}{2} \\
\frac{\overline{A B}}{2}+\frac{\overline{B A}}{2}-\frac{\overline{A A}}{2}-\frac{\overline{B B}}{2} \\
\frac{\overline{A B}}{2}-\frac{\overline{B A}}{2}
\end{array}\right]
$$

$A A, A B, B A$ and $B B$ are genotypic values and $r$ is the reference point of the model, i.e. the midpoint between homozygotes, $a$ gives the additive genotypic value, i.e. half of the difference between two homozygotes, $d$ the dominance genotypic value, i.e. the difference between the heterozygote mean and the midpoint of the homozygotes and finally $i$ denotes the imprinting value, i.e. half the difference between heterozygotes [1].

To detect QTL we performed a genome scan using a linear mixed model fitted by restricted maximum likelihood (REML) with locus $a$, locus $d$ and locus $i$ as fixed class variables [9] and family as a random effect variable to control for the background influences of other loci and shared environmental effects.

Significant genome-wide thresholds for each trait were calculated by 1000 permutations [44]. In addition to these genome-wide thresholds, we chose point-wise LOD scores higher than 1.3 ( $\mathrm{p} \leq 0.05)$. The latter threshold was applied to detect pleiotropic effects when a locus was significant at the genome-wide level for other 
traits [8]. QTL are identified when either the overall LOD (mLOD) or the $a, d, i$ LOD scores exceeded the genome-wide threshold for a given trait.

Our prior work demonstrated that parent-of-origin dependent effects on offspring phenotypes may be caused by either maternal genetic effects or genomic imprinting [22]. Differences in maternal genotype can cause differences between phenotypes of heterozygous offspring and thus cause the same parent-of-origin effect patterns as those caused by genomic imprinting effects. We therefore tested all loci with a significant parent-oforigin effect to determine whether the effect is due to a maternal genetic effect or genomic imprinting. This was achieved by using a mixed model to test whether the parent-of-origin-dependent effect differed significantly between individuals reared by homozygous versus heterozygous mothers [1].

Finally, the proportion of phenotypic variance explained by a locus was calculated by dividing the genotypic variance $(\mathrm{Vg})$ by the phenotypic variance $(\mathrm{Vp})$ given that REML does not compute sums of squares and the corresponding $R^{2}$, following Hager et al. [17]. For additive effects, the explained phenotypic variance by each QTL effect is $\left(1 / 2 a^{2} / \mathrm{Vp}\right) \times 100$; for dominance effects this is given by $\left(1 / 4 d^{2} / \mathrm{Vp}\right) \times 100$, and for imprinting effects by $(1 / 2 \mathrm{i} 2 / \mathrm{Vp}) \times 100$.

We denote QTL as Mc, for mouse chromosome, followed by the number identifying the chromosome and the number of a particular locus on a chromosome.

\section{Additional files}

Additional file 1: Table S1. Number of individuals for each of the fou genotypes at each of the markers. Frequencies of the four genotypes at each of the markers.

Additional file 2: Table S2. Comparison of $\mathrm{IQTL}$ with regions showing parent-origin specific gene expression in the hypothalamus (POA) and medical prefrontal cortex (mFPC), given by sex and expression pattern from [27].

Additional file 3: Figure S1. Map of reference single nucleotide polymorphisms used in this study.Positions are given in Mb. Bars indicate identified QTL with genome-wide significance.

\section{Competing interests}

The authors declare that they have no competing interests.

\section{Authors' contributions}

ARV, RH and SK conducted the analysis. SK collected pheno- and genotypes. $\mathrm{RH}$, SK and GAB conceived the analysis. All authors contributed to writing the manuscript. All authors read and approved the final manuscript.

\section{Acknowledgments}

ARV was supported by the University of Manchester. $\mathrm{RH}$ was supported by a NERC research fellowship. SK and the project were supported by the Deutsche Forschungsgemeinschaft, Project BR 1285/8, to GAB.

\section{Author details}

'Department for Crop and Animal Sciences, Humboldt-University Berlin, Berlin, Germany. ${ }^{2}$ Computational and Evolutionary Biology, Faculty of Life Sciences, University of Manchester, Manchester M13 9PT, UK.

Received: 8 March 2012 Accepted: 13 July 2012

Published: 20 August 2012

\section{References}

1. Hager R, Cheverud JM, Wolf JB: Change in maternal environment induced by cross-fostering alters genetic and epigenetic effects on complex traits in mice. Proc Roy Soc B 2009, 276:2949-2954.

2. Bartolomei MS, Ferguson-Smith AC: Mammalian genomic imprinting. Cold Spring Harb Perspect Biol 2011, 3:1-17.

3. Kelsey G: Epigenetics and the brain: Transcriptome sequencing reveals new depths to genomic imprinting. Bioessays 2011, 33:362-367.

4. Thorvaldsen $\mathrm{J}$, Duran $\mathrm{KL}$, Bartolomei MS: Deletion of the $\mathrm{H} 19$ differentially methylated domain results in loss of imprinted expression of $\mathrm{H} 19$ and lgf2. Gene Dev 1998, 12:3693-3702.

5. Wood AJ, Oakey RJ: Genomic imprinting in mammals: emerging themes and established theories. PLOS Genet 2006, 2:e147.

6. Wolf JB, Hager R, Cheverud JM: Genomic imprinting effects on complex traits A phenotype-based perspective. Epigenetics 2008, 3:295-299.

7. de Koning DJ, Rattink AP, Harlizius B, van Arendonk JAM, Brascamp EW, Groenen MAM: Genome-wide scan for body composition in pigs reveals important role of imprinting. Proc Natl Acad Sci USA 2000, 97:7947-7950.

8. Cheverud JM, Hager R, Roseman C, Fawcett G, Wang B, Wolf JB: Genomic imprinting effects on adult body composition in mice. Proc Natl Acad Sci USA 2008, 105:4253-4258.

9. Wolf JB, Cheverud JM, Roseman C, Hager R: Genome-wide analysis reveals a complex pattern of genomic imprinting in mice. PLoS Genet 2008, 4:e1000091.

10. Hager R, Cheverud JM, Wolf JB: Genotype dependent responses to levels of sibling competition over maternal resources in mice. Heredity 2012, 108:507-514.

11. Sandor C, Georges M: On the detection of imprinted quantitative trait loci in line crosses: effect of linkage disequilibrium. Genetics 2000, 180:1167-1175.

12. Luedi PP, Hartemink AJ, Jirtle RL: Genome-wide prediction of imprinted murine genes. Genome Res 2005, 15:875-884.

13. Bartolomei MS, Tilghman SM: Genomic imprinting in mammals. Annu Rev Genet 1997, 31:493-525.

14. Bevova MR, Aulchenko YS, Aksu S, Renne U, Brockmann GA: Chromosomewise dissection of the genome of the extremely big mouse line DU6i. Genetics 2006, 172:401-410

15. Champagne FA, Curley JP, Swaney WT, Hasen NS, Keverne EB: Paternal influence on female behavior: the role of Peg3 in exploration, olfaction, and neuroendocrine regulation of maternal behavior of female mice. Behav Neurosci 2009, 123:469-480.

16. Davies W, Isles AR, Wilkinson LS: Imprinted gene expression in the brain. Neurosci Biobehav 2005, 29:421-430

17. Hager R, Cheverud JM, Wolf JB: Relative contribution of additive, dominance, and imprinting effects to phenotypic variation in body size and growth between divergent selection lines of mice. Evolution 2009, 63:1118-1128

18. Cockett NE, Jackson SP, Shay TL, Nielsen D, Moore SS, Steele MR, Barendse W, Green RD, Georges M: Chromosomal localization of the callipyge gene in sheep (Ovis aries) using bovine DNA markers. Proc Natl Acad Sci USA 1994, 91:3019-3023.

19. Smit M, Segers K, Carrascosa LG, Shay T, Baraldi F, Gyapay G, Snowder G, Georges M, Cockett N, Charlier C: Mosaicism of solid gold supports the causality of a noncoding A-to-G transition in the determinism of the callipyge phenotype. Genetics 2003, 163:453-456.

20. Nezer C, Moreau L, Brouwers B, Coppieters W, Detilleux J, Hanset R, Karim L, Kvasz A, Leroy P, Georges M: An imprinted QTL with major effect on muscle mass and fat deposition maps to the IGF2 locus in pigs. Nat Genet 1999, 21:155-156.

21. Neugebauer N, Raeder I, Schild HJ, Zimmer D, Reinsch N: Evidence for parent-of-origin effects on genetic variability of beef traits. J Anim Sci 2010, 88:523-532. 
22. Hager R, Cheverud JM, Wolf JB: Maternal effects as the cause of parent-oforigin effects that mimic genomic imprinting. Genetics 2008, 178:1755-1762.

23. Cox A, Ackert-Bicknell CL, Dumont BL, Ding Y, Bell JT, Brockmann GA, Wergedal JE, Bult C, Paigen B, Flint J, Tsaih SW, Churchill GA, Broman KW: A new standard genetic map for the laboratory mouse. Genetics 2009, 182:1335-1344.

24. Lander ES, Botstein D: Mapping Mendelian factors underlying quantitative traits using RFLP linkage maps. Genetics 1989, 121:185-199.

25. Lawson HA, Cady JE, Partridge C, Wolf JB, Semenkovich CF, Cheverud JM: Genetic effects at pleiotropic loci are context-dependent with consequences for the maintenance of genetic variation in populations. PLoS Genet 2011, 7:e1002256.

26. Wilkins JF, Haig D: What good is genomic imprinting: The function of parent-specific gene expression. Nat Genet Rev 2003, 4:359-368.

27. Gregg C, Zhang J, Weissbourd B, Luo S, Schroth GP, Haig D, Dulac C: Highresoultion analysis of parent-of origin allelic expression in the mouse brain. Science 2010, 329:643-648.

28. Kärst S, Cheng R, Schmitt AO, Yang H, de Villena FPM, Palmer AA, Brockman GA: Genetic determinants for intramuscular fat content and waterholding capacity in mice selected for high muscle mass. Mamm Genome 2011, 22:530-543.

29. Brockmann GA, Kratzsch J, Haley CS, Renne U, Schwerin M, Karle S: Single QTL effects, epistasis, and pleiotropy account for two-thirds of the phenotypic F2 variance of growth and obesity in DU6i x DBA / 2 Mice. Genome Res 2000, 10:1941-1957.

30. Lundstrom K, Andersson A, Hansson I: Effect of the RN gene on technological and sensory meat quality in crossbred pigs with Hampshire as terminal sire. Meat Sci 1996, 42:145-153.

31. Gill CO, Newton KG: Spoilage of vacuum-packaged dark, firm, dry meat at chill temperatures. Appl Environ Microbiol 1979, 37:362-364.

32. Grau FH: Inhibition of the anaerobic growth of brochothrixthermosphacta by lactic-acid. Appl Environ Microbiol 1980, 40:433-436.

33. Gill CO, Newton KG: Effect of actic-acid concentration on growth on meat of gram-negative psychrotrophs from a meatworks. Appl Environ Microbiol 1982, 43:284-288.

34. Milan D, Jeon JT, Looft C, Amarger V, Robic A, Thelander M, Rogel-Gaillard C, Paul S, lanuccelli N, Rask L, Ronne H, Lundstrom K, Reinsch N, Gellin J, Kalm E, LeRoy P, Chardon P, Andersson L: A mutation in PRKAG3 associated with excess glycogen content in pig skeletal muscle. Science 2000, 288:1248-1251.

35. Wagener A, Schmitt AO, Aksu S, Schlote W, Neuschl C, Brockmann GA: Genetic, sex, and diet effects on body weight and obesity in the Berlin Fat Mouse Inbred lines. Physiol Genomics 2006, 27:264-270.

36. Schmitt AO, Bortfeldt R, Neuschl C, Brockmann GA: RANDOMATE: a program for the generation of random mating schemes for small laboratory animals. Mamm Genome 2009, 20:321-325.

37. van Laack R, Kaufman RG: Glycolytic potential of red, soft, exudative pork longissimus muscle. J Anim Sci 1999, 77:2971-2973.

38. Tinsley FC, Taicher GZ, Heiman ML: Evaluation of a quantitative magnetic resonance method for mouse whole body composition analysis. Obesity Res 2004, 12:150-160.

39. Neuschl C, Hantschel C, Wagener A, Schmitt AO, Illig T, Brockmann GA: A unique genetic defect on chromosome 3 is responsible for juvenile obesity in the Berlin Fat Mouse. Int J Obesity 2010, 34:1706-1714.

40. Barham D, Trinder P: Improved color reagent for determination of bloodglucose by oxidase system. Analyst 1972, 97:142-145.

41. Yang H, Ding YM, Hutchins LN, Szatkiewicz J, Bell TA, Paigen BJ, Graber JH, de Villena FP, Churchill GA: A customized and versatile high-density genotyping array for the mouse. Nat Methods 2009, 6:663-U55.

42. Xu Z, Zou F, Vision TJ: Improving quantitative trait loci mapping resolution in experimental crosses by the use of genotypically selected samples. Genetics 2005, 170:401-408.

43. Li J, Jiang T: Computing the minimum recombinant haplotype configuration from incomplete genotype data on a pedigree by integer linear programming. J Comp Biol 2005, 12:719-739.

44. Churchill GA, Doerge RW: Empirical threshold values for quantitative trait mapping. Genetics 1994, 138:963-971.

\section{Submit your next manuscript to BioMed Central and take full advantage of:}

- Convenient online submission

- Thorough peer review

- No space constraints or color figure charges

- Immediate publication on acceptance

- Inclusion in PubMed, CAS, Scopus and Google Scholar

- Research which is freely available for redistribution 